because bone marrow-derived SMCs cannot utilize this promoter in the intima. Conversely, how can we be sure that the intimal cells seen by Wamhoff et al. were not bone marrow derived?

In summary, the work by Hendrix et al. (1) may change our focus from vague notions of phenotypic modulation to studying the response by specific genes to specific stimuli. Science progresses by discoveries that change our paradigms. It remains to be seen whether detailed promoter analyses will lead to new paradigms to classify SMCs; to elucidate whether arterial SMCs of nonvascular origin use the CArG box mechanism to differentiate into vascular SMCs; and to ultimately explain how SMCs respond to injury.

\section{Acknowledgments}

The authors wish to acknowledge support from the NIH (grant 2 RO1 HL26405, entitled "Endothelial Injury in Small Vessels").

Address correspondence to: Stephen M. Schwartz, University of Washington, Department of Pathology, Box 357335, HSB Rm. 1-416, 1959 NE Pacific Street, Seattle, Washington 98195-7335, USA.
Phone: (206) 543-0258; Fax: (206) 5435657; E-mail: steves@u.washington.edu.

1. Hendrix, J.A., et al. 2005. 5' CArG degeneracy in smooth muscle $\alpha$-actin is required for injury-induced gene suppression in vivo. J. Clin. Invest. 115:418-427. doi:10.1172/JCI200522648.

2. Wamhoff, B.R., et al. 2004. A G/C element mediates repression of the SM22alpha promoter within phenotypically modulated smooth muscle cells in experimental atherosclerosis. Circ. Res. 95:981-988.

3. Thyberg, J., Blomgren, K., Roy, J., Tran, P.K., and Hedin, U. 1997. Phenotypic modulation of smooth muscle cells after arterial injury is associated with changes in the distribution of laminin and fibronectin. J. Histochem. Cytochem. 45:837-846.

4. Campbell, J.H., and Campbell, G.R. 1994. The role of smooth muscle cells in atherosclerosis. Curr. Opin. Lipidol. 5:323-330.

5. Miano, J.M. 2004. Channeling to myocardin. Circ. Res. 95:340-342.

6. Wang, D.Z., and Olson, E.N. 2004. Control of smooth muscle development by the myocardin family of transcriptional coactivators. Curr. Opin. Genet. Dev. 14:558-566

7. Miano, J.M. 2003. Serum response factor: toggling between disparate programs of gene expression. J. Mol. Cell. Cardiol. 35:577-593.

8. Chen, Y.H., Layne, M.D., Watanabe, M., Yet, S.F., and Perrella, M.A. 2001. Upstream stimulatory factors regulate aortic preferentially expressed gene-1 expression in vascular smooth muscle cells. J. Biol. Chem. 276:47658-47663.

9. Aikawa, M., Yamaguchi, H., Yazaki, Y., and Nagai, R. 1995. Smooth muscle phenotypes in developing and atherosclerotic human arteries demonstrated by myosin expression. J. Atheroscler. Thromb. 2:14-23.
10. Mulvihill, E.R., et al. 2004. Atherosclerotic plaque smooth muscle cells have a distinct phenotype. Arterioscler. Thromb. Vasc. Biol. 24:1283-1289.

11. Frid, M.G., Dempsey, E.C., Durmowicz, A.G., and Stenmark, K.R. 1997. Smooth muscle cell heterogeneity in pulmonary and systemic vessels. Importance in vascular disease. Arterioscler. Thromb. Vasc. Biol. 17:1203-1209.

12. Davie, N.J., et al. 2004. Hypoxia-induced pulmonary artery adventitial remodeling and neovascularization: contribution of progenitor cells. Am. J. Physiol. Lung Cell Mol. Physiol. 286:L668-L678.

13. Gabbiani, G. 2003. The myofibroblast in wound healing and fibrocontractive diseases. J. Pathol. 200:500-503.

14. deRuiter, M.C., et al. 1997. Embryonic endothelial cells transdifferentiate into mesenchymal cells expressing smooth muscle actins in vivo and in vitro. Circ. Res. 80:444-451.

15. Sata, M., et al. 2002. Hematopoietic stem cells differentiate into vascular cells that participate in the pathogenesis of atherosclerosis. Nat. Med. 8:403-409.

16. Hu, Y., et al. 2004. Abundant progenitor cells in the adventitia contribute to atherosclerosis of vein grafts in ApoE-deficient mice. J. Clin. Invest. 113:1258-1265. doi:10.1172/JCI200419628.

17. Hillebrands, J.L., Klatter, F.A., and Rozing, J. 2003. Origin of vascular smooth muscle cells and the role of circulating stem cells in transplant arteriosclerosis. Arterioscler. Thromb. Vasc. Biol. 23:380-387.

18. Dor, Y., Djonov, V., and Keshet, E. 2003. Induction of vascular networks in adult organs: implications to proangiogenic therapy. Ann. N. Y. Acad. Sci. 995:208-216

19. Dietrich, H., et al. 2000. Rapid development of vein graft atheroma in ApoE-deficient mice. Am. J. Pathol. 157:659-669.

\title{
Birth pangs: the stressful origins of lymphocytes
}

\section{Shiv Pillai}

Center for Cancer Research, Massachusetts General Hospital and Harvard Medical School, Boston, Massachusetts, USA.

\begin{abstract}
Inositol-requiring enzyme 1 (IRE1) is a transmembrane protein that signals from the ER and contributes to the generation of an active spliced form of the transcriptional regulator X-box-binding protein 1 (XBP1). XBP1 is required for the terminal differentiation of $B$ lymphocytes into plasma cells, and IRE1 also participates in this differentiation event. A study in this issue of the JCI reveals, quite unexpectedly, that IRE1 is also required early in B lymphocyte development for the induction of the machinery that mediates Ig gene rearrangement (see the related article beginning on page 268).
\end{abstract}

Commitment of a common lymphoid progenitor to the B lineage requires the initia-

Nonstandard abbreviations used: ATF6, activating transcription factor 6; CHOP, C/EBP-homologous protein; eIF2 $\alpha$, eukaryotic translation initiation factor $2 \alpha$; IRE1, inositol-requiring enzyme 1 ; PERK, double-stranded RNA-activated protein kinase-like ER kinase; TdT, terminal deoxynucleotidyl transferase; UPR, unfolded protein response; XBP1, X-box-binding protein 1.

Conflict of interest: The author has declared that no conflict of interest exists.

Citation for this article: J. Clin. Invest. 115:224-227 (2005). doi:10.1172/JCI200524238. tion of Ig gene rearrangement. After a B cell encounters and responds to antigen, it eventually differentiates into an antibodysecreting plasma cell. It has become apparent over the past few years that events in the ER provide important cues for the differentiation of B cells into plasma cells. A role for the ER as a source of signals that drive early events in B cell development is now beginning to emerge.

A little over a decade ago, an intriguing and novel intracellular signaling pathway was described in budding yeast $(1,2)$.
Misfolded proteins in the ER were shown to activate an integral membrane ER resident protein kinase called inositol-requiring enzyme 1 (IRE1) and thus induce the synthesis of chaperone genes that assist in the retention of misfolded proteins in the ER and in the facilitation of their proper folding and assembly. IRE1 contains a lumenal stress-sensor domain, a hydrophobic transmembrane anchor sequence, and cytosolic kinase and endoribonuclease domains (Figure 1). Oligomerization of IRE1 induced by misfolded proteins in the ER lumen results in the activation of IRE1 kinase activity, and the consequent autophosphorylation-dependent activation of the adjacent endoribonuclease domain (3). This latter domain catalyzes an unusual splicing event that generates a shorter spliced form of an mRNA encoding a transcription factor called HAC1. This in turn orchestrates the transcriptional activation 


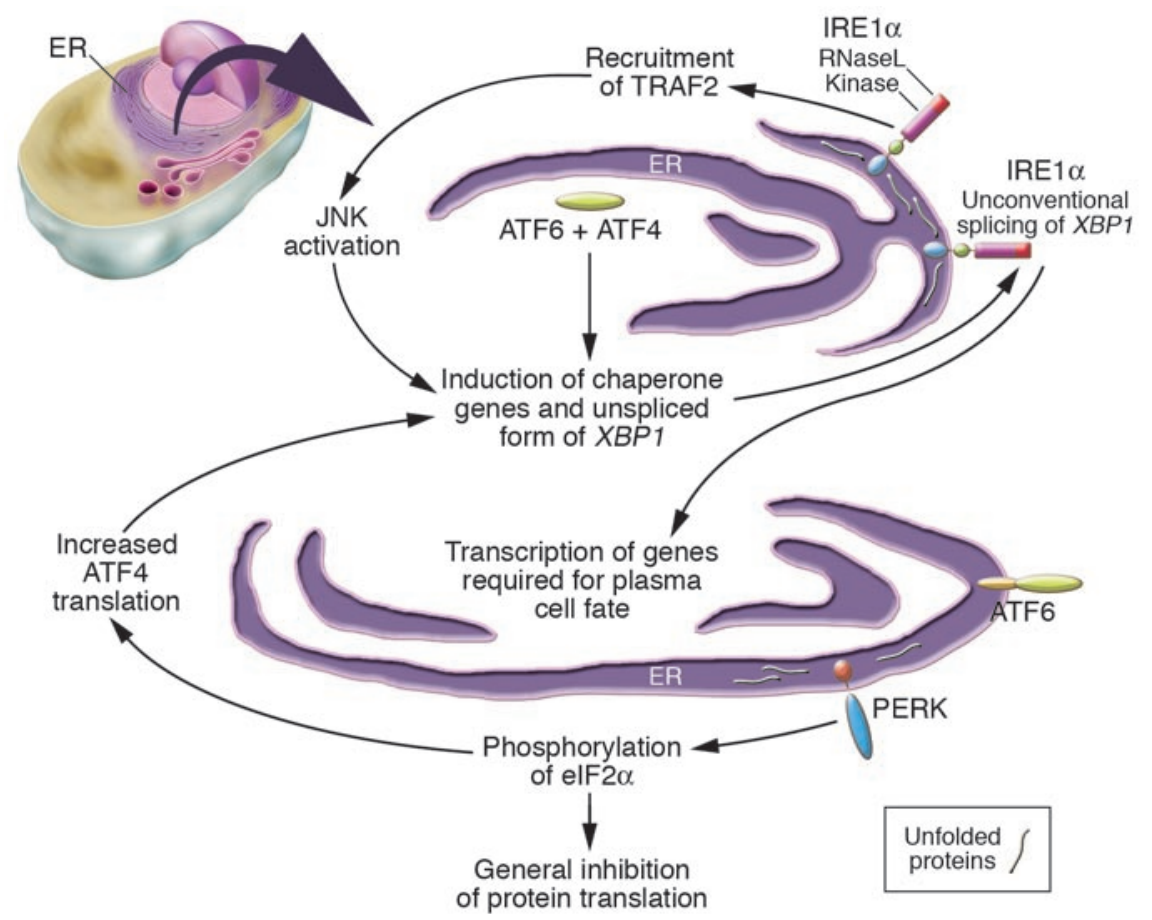

Figure 1

Multiple sensors initiate the UPR in vertebrates. IRE1 $\alpha$ and PERK are integral-membrane ER kinases whose lumenal domains are triggered by misfolded proteins in the ER. IRE $1 \alpha$ and its yeast homolog, IRE1, contain a lumenal stress-sensing domain (blue) as well as cytosolic kinase (magenta) and endoribonuclease (RNaseL, red) domains. ATF6 is another stress sensor, which is cleaved in response to stress to yield a fragment (green) that is transported to the nucleus. Both ATF6 and Blimp-1 (not shown) may contribute to the transcriptional induction of $X B P 1$. Very little is understood as to how IRE1 $\alpha$, a kinase that is activated by unfolded proteins in the ER, contributes to the induction of Rag1, Rag2, and $T d T$ to initiate and sustain V(D)J recombination during early $\mathrm{B}$ cell development.

of a battery of target genes that include many ER chaperones and enzymes that facilitate protein folding. This prototypic stress-regulated signaling pathway is known as the unfolded protein response (UPR) or the ER stress pathway. A number of different causes of ER stress can result in enhanced protein misfolding. These include disordered calcium homeostasis, viral infection, heat shock, and nutrient deprivation, to name a few $(4,5)$. Apart from the physiological and developmental roles of the ER stress pathway, some of which are discussed below, there is growing evidence for its involvement in the pathogenesis of a number of clinical conditions (5-9).

\section{Sensors of the UPR in vertebrates}

In vertebrates, ER stress is monitored by 3 major sensors (Figure 1). These include IRE1 and double-stranded RNA-activated protein kinase-like ER kinase (PERK), which are integral membrane proteins located in the ER, in addition to activating transcription factor 6 (ATF6), which is a type II inte- gral membrane ER protein that contains a C-terminal lumenal domain and can release a cytosolically-oriented $\mathrm{N}$-terminal basic leucine zipper-containing transcription factor in situations of ER stress $(4,10)$. Murine IRE $1 \alpha$ and $\beta$ (the $\alpha$ isoform is ubiquitous while $\beta$ is restricted to the gut) and PERK contain very similar lumenal stress-sensing domains. These lumenal domains are normally physically associated with the ER chaperone, Bip. However, misfolded proteins associate with Bip, causing it to be released from IRE1 and PERK. The release of Bip results in the oligomerization and activation of these kinases. The cytoplasmic regions of murine IRE1 proteins contain kinase and endoribonuclease domains much like their yeast counterparts, and IRE1 in vertebrates lies upstream of X-box-binding protein 1 (XBP1), the vertebrate homolog of HAC1. PERK kinase activity results in the phosphorylation of the $\alpha$ subunit of eukaryotic translation initiation factor 2 $(\mathrm{eIF} 2 \alpha)$ in the cytosol (11). This results in a general inhibition of protein translation, thereby indirectly inhibiting the accumulation of toxic misfolded proteins (Figure 1). However, phosphorylated eIF $2 \alpha$ also mediates the specific and selective enhancement of the translation of ATF4. Transcription of a number of UPR-regulated genes, such as C/EBP-homologous protein (CHOP) and Bip, is enhanced by ATF4. Stress also facilitates the egress of unprocessed ATF6 from the ER to the Golgi, and this in turn results in the sequential cleavage in the Golgi of ATF6 by the site- 1 and site- 2 proteases, releasing active ATF6. ATF6 in collaboration with B lymphocyte-induced maturation protein 1 (Blimp-1; which represses the PAX5 inhibitor), enhances the transcription of $X B P 1$, which is initially generated as a larger, incompletely spliced RNA. IRE1 $\alpha$ is largely localized to the inner nuclear membrane (10). This protein becomes an active kinase during conditions of stress, and its endoribonuclease activity mediates a unique splicing event generating a distinct shorter, functional form of XBP1. In addition, activated IRE1 can recruit TNF receptor-associated factor 2 (TRAF2), which in turn may contribute to the activation of JNK. It is believed that JNK activation and induction of the CHOP transcription factor as well as the activation of caspases 7 and 12 may all contribute to the apoptotic death of severely stressed cells.

\section{B lymphocyte development and ER stress signaling}

During B lymphocyte development, initially pro-B cells rearrange the Ig heavy chain locus in a step-wise fashion (Figure 2). The Rag1 and Rag2 proteins are lymphoid-specific proteins that mediate the site-specific recognition and cleavage of DNA during $V(D) J$ recombination. In addition to Rag1 and Rag2, terminal deoxynucleotidyl transferase (TdT) is another lymphoid-specific factor that contributes to the generation of diverse antibodies. Approximately 1 in 3 pro-B cells eventually makes an inframe rearrangement at the Ig heavy chain locus that is capable of generating the $\mu$ heavy chain protein. A portion of the membrane form of the $\mu$ heavy chain assembles with surrogate light chains to generate a structure known as the pre-B cell receptor, which drives further B cell development. Following the rearrangement of both Ig heavy and light chain genes and the synthesis of fully assembled immunoglobulins, pre-B cells differentiate further into immature B cells and emerge in the periphery as naive $B$ cells. The activation of naive $B$ cells by either $T$ cell-independent or $T$ 


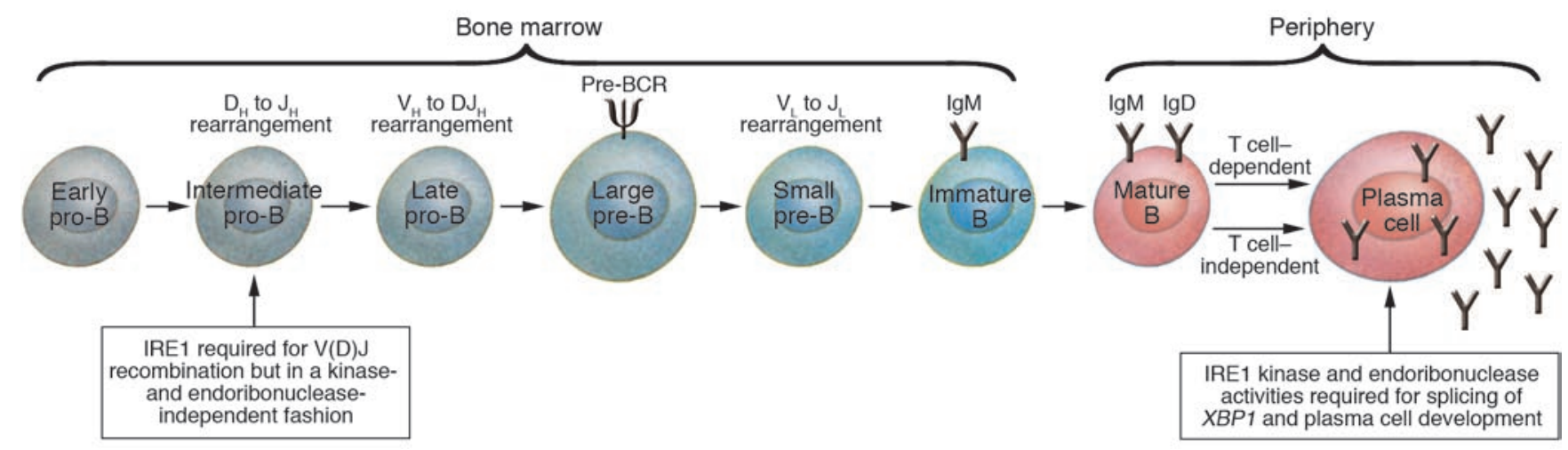

Figure 2

A simplified overview of B cell development. Differentiation is initiated in the bone marrow in an antigen-independent manner and is completed in the periphery in response to antigenic challenge. Rearrangement of the Ig heavy chain is initiated in pro- $B$ cells and involves sequential $D_{H}$ to $\mathrm{J}_{H}$ and $\mathrm{V}_{\mathrm{H}}$ to $D \mathrm{~J}_{H}$ rearrangements. Once light chain rearrangement is completed, $\mathrm{B}$ cells emigrate to the periphery and give rise to multiple peripheral lineages (not shown). Peripheral B cells activated by either T cell-dependent or T cell-independent antigens differentiate into plasma cells. In this issue, Zhang et al. (15) demonstrate that IRE1 is required for V(D)J recombination early in B cell development, but in a kinase- and endoribonuclease-independent fashion. IRE1 kinase and endoribonuclease activities are required for the splicing of XBP1 and plasma cell development. Pre-BCR, pre-B cell receptor.

cell-dependent antigens results in their eventual differentiation into specialized antibody-secreting cells known as plasma cells (Figure 2).

Apart from its role in cellular adaptation, the ER stress response also participates in developmental decisions in vertebrates as well as invertebrates $(12,13)$. XBP1 is critical for the development of plasma cells, and it contributes to the expression not only of ER proteins but also of many genes that contribute to the phenotypic changes that characterize secretory cells, such as expansion of the ER and induction of chaperones, and enzymes such as protein disulfide isomerases (14). In this issue of the JCI, Zhang et al. (15) use a gene inactivation approach to show that IRE $1 \alpha$ is required for the development of plasma cells. Since IRE $1 \alpha$ lies upstream of XBP1, this clarifies that the developmental role of XBP1 is indeed linked to an ER-signaling event. An intriguing and extremely novel finding in this study is the link observed between IRE $1 \alpha$ and $V(D) J$ recombination during $B$ cell lymphopoiesis. In the absence of IRE1 $\alpha$, the accumulation at the pro-B cell stage of mRNAs for the lymphoid-specific proteins that mediate $\mathrm{V}(\mathrm{D}) \mathrm{J}$ recombination - namely Rag1, Rag2, and TdT - is significantly compromised. None of the obvious suspects - genes that are known to participate in early B cell commitment and developmental progression, such as Ikaros, PU.1, Pax5, EBF, or E2A, were found to be absent or to suffer reduced levels of expression at this stage of development in the absence of IRE $1 \alpha$. In contrast to IRE1 $\alpha$, PERK and $\mathrm{XBP} 1$ were not found to be required early in B cell development.

One possible link that might have been considered to exist between the UPR and early B cell development is the phenomenon of heavy chain toxicity. In pre-B cells, a considerable amount of the $\mu$ heavy chain protein is misfolded or is incompletely assembled with components of the pre-B cell receptor and is recognized in the ER by specific chaperones, such as Bip, calnexin, and calreticulin. These chaperones retain unassembled and misfolded proteins in the $\mathrm{ER}$, providing these proteins with the opportunity to fold and assemble properly or helping to facilitate their retro-translocation into the cytosol and degradation in proteasomes (16). The notion that Ig heavy chain proteins in the absence of a regular light chain may be toxic to cells (heavy chain toxicity) was first enunciated by George Kohler (17) and is probably linked to signaling resulting in apoptosis that is induced by misfolded proteins in the ER. In has been suggested that pre-B cells are somehow protected from heavy chain toxicity (18), and it might well be that the UPR helps pre-B cells adapt to ER stress and thus permits their survival.

Some of the findings presented by Zhang et al. (15), however, fail to support a connection between the UPR and protection from heavy chain toxicity Although the cytoplasmic tail of IRE1 $\alpha$ is required for the expression of Rag and $T d T$, the catalytic activities of the cytosolic kinase and endoribonuclease domains of IRE $1 \alpha$ are not. This suggests that this particular developmental function of IRE1 $\alpha$ is unrelated to its activation by misfolded proteins in the ER. The possibility that TRAF2 contributes to the IRE1 $\alpha$-dependent control of early B cell development has been considered by Zhang et al., but since the recruitment of this adaptor by IRE1 $\alpha$ depends on the latter's kinase activity (19), some other unknown kinase-independent biochemical function of IRE1 $\alpha$ may need to be invoked. It should be emphasized that while IRE1 $\alpha$ may be important during the development of $\mathrm{B}$ and possibly $\mathrm{T}$ cells, little data exists to link the UPR itself to IRE1 $\alpha$ 's role early in B cell ontogeny. IRE $1 \alpha$ has already been shown to localize to the inner nuclear membrane, and this might facilitate the ability of IRE1 $\alpha$ to regulate gene expression early in B cell development. As Zhang et al. point out, IRE1 in yeast associates with a specific transcriptional activator, and in developing lymphocytes, the vertebrate homolog may possibly function as a molecular scaffold, somehow facilitating the transcription of Rag genes and TdT. There is a growing appreciation of the fact that many genes expressed by lymphocytes are sequestered in the periphery of the nucleus when they are silent (20). The possibility that IRE1 $\alpha$ might contribute in some direct or indirect way to the physical localization of the Rag locus and TdT during B cell development probably merits further exploration.

Address correspondence to: Shiv Pillai, Center for Cancer Research, Massachusetts 
General Hospital Building 149, 13th Street, Charlestown Navy Yard, Boston, Massachusetts 02129, USA. Phone: (617) 726-5619; Fax: (617) 724-9648; E-mail: pillai@helix.mgh.harvard.edu.

1. Mori, K., Ma, W., Gething, M.J., and Sambrook, J. 1993. A transmembrane protein with a cdc2+/ CDC28-related kinase activity is required for signaling from the ER to the nucleus. Cell. 74:743-756.

2. Cox, J.S., Shamu, C.E., and Walter, P. 1993. Transcriptional induction of genes encoding endoplasmic reticulum resident proteins requires a transmembrane protein kinase. Cell. 73:1197-1206.

3. Shamu, C.E., and Walter, P. 1996. Oligomerization and phosphorylation of the Ire $1 \mathrm{p}$ kinase during intracellular signaling from the endoplasmic reticulum to the nucleus. EMBO J. 15:3028-3039.

4. Sitia, R., and Braakman, I. 2003. Quality control in the endoplasmic reticulum protein factory. Nature. 426:891-894

5. Kaufman, R.J. 2002. Orchestrating the unfolded protein response in health and disease. J. Clin. Invest. 110:1389-1398. doi:10.1172/JCI200216886.
6. Imaizumi, K., et al. 2001. The unfolded protein response and Alzheimer's disease. Biochim. Biophys. Acta. 1536:85-96.

7. Ryu, E.J., et al. 2002. Endoplasmic reticulum stress and the unfolded protein response in cellular models of Parkinson's disease. J. Neurosci. 22:10690-10698.

8. Ozcan, U., et al. 2004. Endoplasmic reticulum stress links obesity, insulin action, and type 2 diabetes. Science. 306:457-461.

9. Aridor, M., and Balch, W.E. 1999. Integration of endoplasmic reticulum signaling in health and disease. Nat. Med. 5:745-751.

10. Lee, K., et al. 2002. IRE1-mediated unconventional mRNA splicing and S2P-mediated ATF6 cleavage merge to regulate XBP1 in signaling the unfolded protein response. Genes Dev. 16:452-466.

11. Shi, Y., et al. 1998. Identification and characterization of pancreatic eukaryotic initiation factor 2 alpha-subunit kinase, PEK, involved in translational control. Mol. Cell. Biol. 18:7499-7509.

12. Shen, X., et al. 2001. Complementary signaling pathways regulate the unfolded protein response and are required for C. elegans development. Cell. 107:893-903.

13. Reimold, A.M., et al. 2001. Plasma cell differentiation requires the transcription factor XBP-1.
Nature. 412:300-307.

14. Shaffer, A.L., et al. 2004. XBP1, downstream of Blimp-1, expands the secretory apparatus and other organelles, and increases protein synthesis in plasma cell differentiation. Immunity. 21:81-93.

15. Zhang, K., et al. 2005. The unfolded protein response sensor IRE $1 \alpha$ is required at 2 distinct steps in B cell lymphopoiesis. J. Clin. Invest. 115:268-281. doi:10.1172/JCI200521848.

16. Ho, S., Chaudhuri, S., Bacchawat, A.K., McDonald, K., and Pillai, S. 2000. Accelerated proteasomal degradation of membrane immunoglobulin heavy chains. J. Immunol. 164:4713-4719.

17. Kohler, G. 1980. Immunoglobulin chain loss in hybridoma lines. Proc. Natl. Acad. Sci. U. S. A. 77:2197-2199.

18. Haas, I.G., and Wabl, M. 1984. Immunoglobulin heavy chain toxicity in plasma cells is neutralized by fusion to pre-B cells. Proc. Natl. Acad. Sci. U. S. A. 81:7185-7188.

19. Urano, F., et al. 2000. Coupling of stress in the ER to activation of JNK protein kinases by transmembrane protein kinase IRE1. Science. 287:664-666.

20. Kosak, S.T., et al. 2002. Subnuclear compartmentalization of immunoglobulin loci during lymphocyte development. Science. 296:158-162.

\section{Rab27a: a new face in $\beta$ cell metabolism-secretion coupling}

Toru Aizawa ${ }^{1}$ and Mitsuhisa Komatsu²

${ }^{1}$ Center for Health, Safety, and Environmental Management and ${ }^{2}$ Graduate School of Medicine, Department of Aging Medicine and Geriatrics, Shinshu University, Matsumoto, Japan.

In pancreatic $\beta$ cells, not only insulin exocytosis per se, but translocation of $\beta$ granules toward the plasma membrane - an event upstream of exocytosis - are under the control of glucose. However, the molecular basis of this translocation has been poorly understood. Rab27a-mediated translocation of glucose-induced $\beta$ granules is reported in this issue of the JCI (see the related article beginning on page 388 ). Rab27a or its effector molecule may constitute a novel pharmacological target because potentiation of the Rab27a pathway is expected to restore $\beta$ cell glucose competency in patients with diabetes mellitus.

Insulin secretion by the pancreatic $\beta$ cell is tightly regulated by nutrients, especially by glucose. Selective impairment of glucose-stimulated insulin secretion (GSIS) is a salient feature of $\beta$ cell dysfunction in patients with type 2 diabetes mellitus. For

Nonstandard abbreviations used: $\left[\mathrm{Ca}^{2+}\right]_{i}$, intracellular $\mathrm{Ca}^{2+}$ concentration; GIP, glucose-dependent insulinotropic peptide; GLP1, glucagon-like peptide 1; GSIS, glucose-stimulated insulin secretion; $\mathrm{K}_{\mathrm{ATP}}$, ATP-sensitive $\mathrm{K}^{+}$; LC-CoA, long-chain CoA; RP, reserve pool; $\mathrm{RRP}$, readily releasable pool; $\mathrm{SU}$, sulfonylurea; TCA, tricarboxylic acid; t-SNARE, target membrane soluble $\mathrm{N}$-ethylmaleimide-sensitive factor attachment protein receptor; v-SNARE, vesicle membrane SNARE.

Conflict of interest: The authors have declared that no conflict of interest exists.

Citation for this article: J. Clin. Invest. 115:227-230 (2005). doi:10.1172/JCI200524269.
GSIS to occur, insulin-containing $\beta$ granules are transported to the vicinity of the plasma membrane, docked, and primed, so that they become readily releasable upon elevation of intracellular $\mathrm{Ca}^{2+}$ concentration $\left(\left[\mathrm{Ca}^{2+}\right]_{\mathrm{i}}\right)$ (Figure 1). The molecular basis for glucose stimulation of $\beta$ granule docking and/or replenishment of the readily releasable pool of granules is now at least in part clarified in this issue of the JCI (1).

\section{Mechanism of GSIS}

Glucose metabolism in the $\beta$ cell activates ATP-sensitive $\mathrm{K}^{+}\left(\mathrm{K}_{\text {ATP }}\right)$ channel-dependent and -independent signaling pathways (Figure 1). Mitochondrial metabolism is the key event in both pathways. Pyruvate, a product of glycolysis, enters the tricarboxylic acid
(TCA) cycle, and ATP is then generated. At the same time, cytosolic flux of the TCA cycle intermediate(s) (2) and/or activation of acetyl-CoA carboxylase (3) and activation of phospholipase C (4) take place. Anaplerosis (2), carboxylation of pyruvate to oxaloacetate and its entry to the TCA cycle - rather than entry of pyruvate into the TCA cycle via decarboxylation to acetyl-CoA - is critical for all of these outcomes (5).

The $\mathrm{K}_{\text {ATP }}$ channel-dependent events (Figure 1A) have been well characterized (6), and they include: (a) elevation of cytosolic ATP/ADP following ATP generation; (b) closure of the $\mathrm{K}_{\text {ATP }}$ channels; (c) membrane depolarization; (d) opening of L-type voltage-dependent $\mathrm{Ca}^{2+}$ channels; (e) $\mathrm{Ca}^{2+}$ influx; (f) elevation of $\left[\mathrm{Ca}^{2+}\right]_{i}$; and eventually $(\mathrm{g})$ triggering of exocytosis, i.e., fusion of $\beta$ granules in the readily releasable pool (RRP) and the plasma membrane. It should be noted that $\beta$ granules in the cell do not constitute a uniform population. A majority of them remain inside the cell and cannot immediately be releasable, thus forming a reserve pool (RP), whereas a small portion of them constitute the RRP, which is available for immediate secretion. 